\title{
Impact of nine chronic conditions for US adults aged 65 years and older: an application of a hybrid estimator of quality-adjusted life years throughout remainder of lifetime
}

\author{
Haomiao Jia ${ }^{1}$ Erica I. Lubetkin ${ }^{2}$
}

Accepted: 7 January 2016/Published online: 18 January 2016

(c) Springer International Publishing Switzerland 2016

\begin{abstract}
Purpose To estimate quality-adjusted life years (QALY) loss due to each of the following nine chronic conditionsdepression, diabetes mellitus, hypertension, heart disease, stroke, emphysema, asthma, arthritis, and cancer.

Methods We ascertained respondents' health-related quality of life scores and mortality status from the 2005 to 2008 National Health and Nutrition Examination Survey (NHANES) with mortality follow-up data through December 31, 2011. We included respondents aged 65 years and older $(n=2380)$. A hybrid estimator was used to calculate QALY from two parts: QALY during the follow-up period and QALY beyond the follow-up period. We calculated QALY by each of the nine chronic conditions.

Results For persons aged 65 and older, QALY throughout the reminder of lifetime was 12.3 years. After adjusting for age- and sex-related differences, depression had an associated 8.2 years of QALY loss; diabetes, 5.6 years; hypertension, 2.5 years; heart disease, 5.4 years; stroke, 6.4 years; emphysema, 8.0 years; asthma, 4.8 years; arthritis, 0.3 years; and cancer, 2.5 years. Compared to persons without any chronic conditions, persons with one condition had an associated 4.7 years of QALY loss;
\end{abstract}

Haomiao Jia

hj2198@columbia.edu

1 Department of Biostatistics, Mailman School of Public Health and School of Nursing, Columbia University, 617 West 168th Street, New York, NY 10032, USA

2 Department of Community Health and Social Medicine, Sophie Davis School of Biomedical Education/CUNY Medical School, New York, NY 10031, USA persons with two conditions, 7.9 years; and persons with three or more conditions, 10.8 years.

Conclusions This study presents a QALY estimator for respondents in the NHANES-Linked Mortality File and demonstrates the utility of this method to other follow-up data. Continued application of our method would enable the burden of disease to be compared for a range of health conditions and risk factors in the ongoing effort to improve population health.

Keywords Quality-adjusted life year (QALY) · Healthrelated quality of life (HRQOL) · Chronic conditions . Burden of disease

\section{Introduction}

The health impact of many chronic conditions and risk factors includes both fatal and nonfatal outcomes [1]. For example, diabetes mellitus has been associated with increased mortality [2] and increased risk of disability or deteriorating health-related quality of life (HRQOL) [3]. It would be particularly useful to quantify the lifetime overall burden of a disease or a risk factor in a single value index that reflects all aspects of health, including nonfatal illness and mortality outcomes $[4,5]$. Such analyses will make it possible to enable the direct comparison of the overall burden attributed to different diseases and risk factors [5, $6]$. Several indices have been developed and used for this purpose [7-9]. One of the most widely used indices is disability-adjusted life years (DALY) [8]. It is calculated as the sum of year of life lost (YLL) and years lived in disability (YLD), so this method treats losses to mortality and to morbidity equally and treats the losses at different ages equally. Another widely used index is disability-free life 
expectancy (DFLE), which is a modification of life expectancy [9]. In a period life table, which is commonly used to calculate life expectancy, the number of life years in an age interval for a stationary population cohort is multiplied by the proportion of persons in that age interval who are free of disability. Therefore, DFLE calculation relies on the life table method's stationarity assumption that the age-specific hazard rate (i.e., probability of dying in each age interval) is constant over time [10].

Quality-adjusted life years (QALY) is a health outcome measure that weights life years lived with preference-based health-related quality of life (HRQOL) scores [7]. Preference-based HRQOL, also called health utility value, is a summary score that assesses the values of one health state vs. another state. The health utility value is anchored at 0 for death and 1 for perfect health [11], so 1 year lived in a reduced health state of utility value of 0.5 is equal to 0.5 QALYs, the same as lived one half year in perfect health. The mean QALY throughout the remainder of the lifetime is calculated as [12]:

$\mathrm{QALY}=\int_{0}^{\infty} q(t) S(t) \mathrm{d} t$,

where $q(t)$ is the HRQOL score at time $t$ and $S(t)$ is the proportion surviving to time $t$. The proportion of the population at the start of the cohort is assumed to be 1 (i.e., $S(0)=1)$.

Because QALY uses the health utility value to weight years of life lived, one of the advantages of QALY over DALY and DFLE is that it can be used for calculating the economic costs of a condition or a risk factor and for analyzing the cost-effectiveness of alternative treatments, intervention programs, and health policies $[5,6,12]$. However, calculation of mean QALY can be difficult due to censoring $[13,14]$. It requires extrapolating quality-adjusted survival time beyond the time of the last death occurred [15]. Many investigators instead have calculated mean QALYs to a chosen time point, such the end of the study $[13,14]$. Because the follow-up time of longitudinal data is typically short and most participants do not die during the follow-up, this method does not measure the lifetime health outcome.

Many studies have estimated the impact of some chronic diseases and risk factors on the mean QALY starting from a given age to the remainder of the lifetime for a group of similarly aged persons, called quality-adjusted life expectancy (QALE) [12, 16-18]. By combining the period life table and age-specific HRQOL score, the calculation of QALE is similar to the calculation of DFLE. The number of life years in each age interval for a stationary population cohort in a life table is multiplied by the average HRQOL in that age interval [12]. The main advantage of this method is its ability to use cross-sectional data because it uses estimates of mortality rates and mean HRQOL scores for each age interval [12, 16-18]. However, obtaining robust and unbiased estimates of mortality rates for the target population (such as diseased persons) is difficult since the validity and reliability of these estimates depend on the data used for estimation and model assumptions [17, 18]. Additionally, this method requires a much larger sample size to obtain acceptable precision of estimation, usually tens of thousands [16-18]. Therefore, this method cannot be used for the calculation of burden of disease for many chronic conditions and risk factors due to these limitations.

It is more common to compare participants' QALY between the intervention group and the control group in a randomized controlled trial or between the diseased persons and the nondiseased persons in a population-based cohort [19-21]. These comparisons of QALY are between two groups of participants of different ages and probably of different sociodemographic characteristics and comorbidities. Therefore, mean QALY cannot be calculated using the same life table method for the calculation of QALE.

The main goals of the current study are to introduce a new estimator for QALY during the remainder of lifetime and to demonstrate the utility of this method by estimating the impact on QALY for nine chronic conditions for US adults aged 65 years and older. Six of these nine conditions were chosen on the basis of their inclusion in other national data sets (i.e., depression, diabetes mellitus, hypertension, heart disease, stroke, and asthma) while the other three conditions were chosen on the basis of prevalence among the elderly (arthritis) [22], being a leading cause of death in the USA (cancer) [23] and having a high mortality rate (emphysema) [24]. We also estimated the impact on ageadjusted HRQOL score and mortality rate for each of the nine conditions.

\section{Materials and methods}

We ascertained respondents' HRQOL scores and mortality status from the 2005 to $2008 \mathrm{http}: / / w w w . c d c . g o v / \mathrm{nchs} /$ nhanes.htm National Health and Nutrition Examination Survey (NHANES)-Linked Mortality File [25, 26]. The NHANES is an ongoing survey of random samples from the noninstitutionalized civilian population of the USA to obtain national statistics on health and nutritional status [25]. With the use of the design weight and adjustment for noncoverage and nonresponse, the distribution of respondents was representative of that of the US general population [25]. The NHANES-Linked Mortality File was created by the National Center for Health Statistics (NCHS) by linking the NHANES respondents to the 
National Death Index (NDI) [26]. The respondents in this analysis had mortality follow-up through December 31, 2011. We included only respondents aged 65 years and older, yielding a total sample size of 2380 .

The NHANES included a questionnaire which asks respondents to rank their general health from 1 (excellent) to 5 (poor) and to report numbers of their physically unhealthy days, mentally unhealthy days, and days with activity limitation during the past 30 days [27]. This study employs a previously published mapping algorithm based on respondents' age and answers to these four questions to obtain values of a frequently used preference-based HRQOL measurement, the EQ-5D index, to calculate QALY [28]. This algorithm provides valid estimates of EQ-5D scores for respondents, and the bias of estimated QALY from these scores has been estimated to be $<1 \%$ of that using the actual EQ-5D questions [29].

The NHANES asked respondents whether or not they had ever been told they had diabetes mellitus, hypertension, asthma, arthritis, heart disease (coronary heart disease, angina, or heart attack), stroke, emphysema, and cancer of any kind by a doctor or other health professional [25]. Women who had diabetes only during pregnancy were excluded. Only persons who reported still having asthma at the time of survey were considered having asthma. Cancer patients who only reported having skin cancer were excluded. Participants' depression status was ascertained from eight-item Patient Health Questionnaire (PHQ-8) depression scale [30]. Current depression is defined as PHQ-8 index $\geq 10$ [30].

\section{Statistical analysis}

Mean QALY throughout the remainder of the lifetime was estimated with a hybrid method which calculated QALY from two parts: QALY during the follow-up period (to December 31, 2011) and QALY beyond the follow-up period (after December 31, 2011).

The QALY during the follow-up period was estimated based on the method by Shen et al. [14]. Let $L$ be the time of the end of follow-up and $0<t_{1} \leq t_{2} \leq \cdots \leq t_{l}<L$ be times when deaths occurred. Suppose $\hat{S}_{\mathrm{KM}}(t)$ is the Kaplan-Meier estimated survival function. We calculate mean QALY at $t_{i}(i=1, \ldots, l), \hat{Q}\left(t_{i}\right)$, for those who died at $t_{i}$; and at time $L$, $\hat{Q}(L)$, for those who were alive at the end of follow-up. QALYs for time period $(0, L]$ was estimated as:

$\left[\sum_{j=1}^{l} \hat{Q}\left(t_{j}\right)\left(\hat{S}_{\mathrm{KM}}\left(t_{j-1}\right)-\hat{S}_{\mathrm{KM}}\left(t_{j}\right)\right)\right]+\hat{Q}(L) \hat{S}_{\mathrm{KM}}\left(t_{l}\right)$,

where $t_{0}=0$ and $S\left(t_{0}\right)=S(0)=1$.

$Q A L Y$ beyond the follow-up period was estimated by extrapolating survival function $S(t)$ beyond time $L$.
However, because the model usually fits data well during the early follow-up but does not fit data well near the end of the follow-up, the model may not extrapolate the survival function well in the tail [15]. Instead, we used the parametric method to estimate total expected life years and the Kaplan-Meier method to estimate life years from time 0 to $L$. We used Weibull model, $S_{p}(t)=\exp \left[-(t / \lambda)^{\beta}\right]$ and the QALYs in the tail was estimated as:

$$
\begin{aligned}
& q(L)\left\{\left[\hat{\lambda} \Gamma\left(1+\frac{1}{\hat{\beta}}\right)\right]\right. \\
& \left.\quad-\left\{\left[\sum_{i=0}^{t_{k-1}} \hat{S}_{K M}\left(t_{i}\right)\left(t_{i+1}-t_{i}\right)\right]+\hat{S}_{K M}\left(t_{k}\right)\left(L-t_{k}\right)\right\}\right\},
\end{aligned}
$$

where $\Gamma(*)$ is the gamma function. We used the mean EQ$5 \mathrm{D}$ index at time $L, q(L)$, for HRQOL estimates beyond the end of follow-up because extrapolation of $q(t)$ outside the data range is usually unreliable.

The QALY loss due to a chronic disease was defined as the difference in QALY for those without the disease and those with the disease [18]. We used a propensity score method to account for the systematic difference in participants' characteristics, such as age and sex, between the diseased and nondisease individuals [31]. This was done by using the inverse probability of selection weight to create a weighted sample in which the distribution of participants' characteristics was independent of disease status.

\section{Results}

The average age of the participants was 73.6 years $(\mathrm{SD}=5.8$ years) at the start of the study (Table 1$)$. Women comprised $56.1 \%$ of the population and white non-Hispanics comprised $84.0 \%$ of the population. Only $8.2 \%$ of participants were black non-Hispanics and $5.5 \%$ were Hispanics. In this population, the mean EQ-5D index was 0.826 (Table 2). About $17.2 \%$ of participants died during the follow-up, yielding a mortality rate of 3.83 deaths per 100 person-years. The mean QALY throughout the remainder of the lifetime was 13.7 years (Table 3 ). Compared to men, women had a better age-adjusted mortality rate (3.10 vs. 4.80) but a worse age-adjusted EQ-5D score (0.807 vs. 0.851). Overall, QALY was significantly higher among women than among men (15.9 vs. 11.5 years) after adjusting for the difference in age between men and women.

\section{Loss due to depression}

For those aged 65 years and older, $3.8 \%$ persons were currently depressed (Table 1). Among those with 
Table 1 Distributions of sample characteristics, 2005-2008 NHANES

\begin{tabular}{|c|c|c|c|}
\hline & $N$ & $\%^{\mathrm{a}}$ & $\overline{\mathrm{SE}^{\mathrm{b}}(\%)}$ \\
\hline Age: mean \pm SD & 2380 & $73.6 \pm 5.8$ & \\
\hline \multicolumn{4}{|l|}{ Age groups } \\
\hline $65-69$ & 523 & 30.2 & 1.5 \\
\hline $70-74$ & 503 & 24.2 & 1.4 \\
\hline $75-79$ & 391 & 19.6 & 1.0 \\
\hline $80-85$ & 511 & 21.0 & 1.5 \\
\hline $85+$ & 116 & 5.0 & 0.7 \\
\hline Women & 1169 & 56.1 & 1.0 \\
\hline \multicolumn{4}{|l|}{ Race } \\
\hline White non-Hispanics & 1521 & 84.0 & 2.0 \\
\hline Black non-Hispanics & 424 & 8.2 & 1.2 \\
\hline Hispanics & 385 & 5.5 & 0.9 \\
\hline Other & 50 & 2.4 & 0.6 \\
\hline Died during follow-up & 453 & 17.2 & 1.2 \\
\hline Depression & 98 & 3.8 & 0.5 \\
\hline Diabetes & 501 & 18.2 & 1.0 \\
\hline Hypertension & 1423 & 59.7 & 1.1 \\
\hline Heart disease $^{c}$ & 532 & 22.5 & 1.2 \\
\hline Stroke & 255 & 10.4 & 1.0 \\
\hline Emphysema & 140 & 6.2 & 0.7 \\
\hline Asthma & 175 & 7.5 & 0.7 \\
\hline Arthritis & 1268 & 55.5 & 1.0 \\
\hline Cancer $^{\mathrm{d}}$ & 401 & 17.6 & 1.0 \\
\hline Conditions: mean $\pm \mathrm{SD}$ & 2380 & $2.0 \pm 1.3$ & \\
\hline \multicolumn{4}{|l|}{ No. of conditions } \\
\hline 0 & 301 & 12.2 & 0.7 \\
\hline 1 & 613 & 26.1 & 1.2 \\
\hline 2 & 671 & 28.6 & 1.0 \\
\hline $3+$ & 795 & 33.1 & 1.4 \\
\hline
\end{tabular}

${ }^{a}$ Weighted percentage, accounted for sampling design, noncoverage, and nonresponse

b Standard error

${ }^{c}$ Coronary heart disease, angina, or heart attack

d Any kind of cancer, excluding skin cancer

depression, the age- and sex-adjusted EQ-5D index was $0.440,0.405$ points lower than those without depression (Table 2). Such a 0.405-point decrease represented a decrease of nearly half $(47.9 \%)$ the EQ-5D index score and was at least 5.4 times as much as the $0.025-0.075$ point decrease for each of the other eight conditions. Depression also had a large impact on mortality rates. The age- and sex-adjusted mortality rate was 6.77 deaths per 100 personyears among depressed persons, 3.20 points higher $(89.6 \%$ higher) than that among persons without depression (3.57 deaths per 100 person-years). The mean QALY throughout the remainder of lifespan for those with depression was 4.6, 9.4 years less than those without depression (14.1 years)
(Table 3). This represented a loss of more than two-thirds $(67.1 \%)$ of QALY due to depression.

The large and significant QALY loss due to depression occurred for both men (7.6-year loss) and women (10.7year loss) and for white non-Hispanics (9.6-year loss) (Fig. 1). The QALY losses for black non-Hispanics and for Hispanics were smaller (4.2- and 2.0-year loss, respectively) and not statistically significant. Women lost more QALY to depression than men did, but men lost a slightly larger percentage of QALY than women did (65.6 vs. $64.3 \%$ ) because men had fewer QALYs. The difference in QALY loss between men and women was not statistically significant.

\section{Loss due to diabetes mellitus}

Nearly one in five $(18.2 \%)$ participants reported that they had ever been told that they had diabetes by a doctor or other health professional. Diabetes had a relatively large impact on mortality and a small impact on HRQOL: 2.32-point increase $(67.8 \%)$ in mortality rate and 0.046 -point decrease $(5.5 \%)$ in the EQ-5D index. The total health loss due to diabetes was large with a loss of 6.3 years in QALY or a loss of $41.2 \%$ QALY. Such diabetes-associated QALY loss occurred in all sex and race/ethnicity subgroups. Women with diabetes lost more QALY (8.6-year loss) than men with diabetes did (4.5year loss) and Hispanics lost more QALY (7.4-year loss) than the other two race/ethnicity subgroups, but these differences were not statistically significant.

\section{Loss due to hypertension, heart disease, or stroke}

In this population, about three in five $(59.7 \%)$ participants had hypertension, one in five $(22.5 \%)$ had heart disease, and one in ten $(10.4 \%)$ had a stroke. The health losses due to hypertension were small for both fatal and nonfatal outcomes. Overall, the impact of hypertension on QALY was small (2.1-year loss) and not statistically significant.

Heart disease had a large impact on mortality and a relatively small impact on HRQOL. Participants with heart disease had a mortality rate that was 2.56-points higher (77.6\%) and an EQ-5D index that was 0.047-points lower $(8.7 \%)$ than participants without heart disease. The total health loss for heart disease was large, with a 6.5-year loss $(40.7 \%)$ in QALY. This loss occurred for women (10.7year loss), white non-Hispanics (7.3-year loss), and black non-Hispanics (6.5-year loss) only. Although elderly men were much more likely to have heart disease than elderly women (29.8 vs. $16.7 \%$ ), men with heart disease lost only 2.3 QALYs and such a loss was not statistically significant.

Compared to those who did not have a stroke, persons who reported having had a stroke had a much higher mortality rate (4.48-point or $131 \%$ higher) and a lower EQ-5D index 
Table 2 Mortality rates and EQ-5D scores by disease status

\begin{tabular}{|c|c|c|c|c|c|c|c|c|c|}
\hline Conditions & $N$ & Mortality $^{a}$ & $\mathrm{SE}^{\mathrm{b}}$ & Increase & SE & EQ-5D ${ }^{c}$ & SE & Decrease & SE \\
\hline Total & 2380 & 3.83 & 0.21 & & & 0.826 & 0.006 & & \\
\hline \multicolumn{10}{|l|}{ Depression } \\
\hline No & 2178 & 3.57 & 0.32 & & & 0.846 & 0.008 & & \\
\hline Yes & 98 & 6.77 & 0.29 & 3.20 & 0.43 & 0.440 & 0.009 & 0.405 & 0.012 \\
\hline \multicolumn{10}{|l|}{ Diabetes } \\
\hline No & 1875 & 3.42 & 0.22 & & & 0.834 & 0.007 & & \\
\hline Yes & 501 & 5.74 & 0.54 & 2.32 & 0.58 & 0.788 & 0.013 & 0.046 & 0.015 \\
\hline \multicolumn{10}{|c|}{ Hypertension } \\
\hline No & 952 & 3.58 & 0.22 & & & 0.856 & 0.006 & & \\
\hline Yes & 1423 & 3.95 & 0.96 & 0.37 & 0.98 & 0.809 & 0.023 & 0.047 & 0.024 \\
\hline \multicolumn{10}{|c|}{ Heart disease $^{\mathrm{d}}$} \\
\hline No & 1830 & 3.30 & 0.22 & & & 0.840 & 0.006 & & \\
\hline Yes & 532 & 5.86 & 1.70 & 2.56 & 1.71 & 0.767 & 0.039 & 0.073 & 0.040 \\
\hline \multicolumn{10}{|l|}{ Stroke } \\
\hline No & 2115 & 3.42 & 0.56 & & & 0.835 & 0.013 & & \\
\hline Yes & 255 & 7.90 & 0.22 & 4.48 & 0.60 & 0.760 & 0.007 & 0.075 & 0.015 \\
\hline \multicolumn{10}{|l|}{ Emphysema } \\
\hline No & 2232 & 3.42 & 0.29 & & & 0.832 & 0.008 & & \\
\hline Yes & 140 & 10.55 & 0.28 & 7.13 & 0.40 & 0.758 & 0.008 & 0.074 & 0.011 \\
\hline \multicolumn{10}{|l|}{ Asthma } \\
\hline No & 2194 & 3.66 & 0.22 & & & 0.833 & 0.007 & & \\
\hline Yes & 175 & 5.69 & 0.67 & 2.03 & 0.71 & 0.760 & 0.017 & 0.073 & 0.018 \\
\hline \multicolumn{10}{|l|}{ Arthritis } \\
\hline No & 1107 & 3.89 & 0.22 & & & 0.854 & 0.006 & & \\
\hline Yes & 1268 & 3.74 & 0.47 & 0.15 & 0.52 & 0.806 & 0.013 & 0.048 & 0.015 \\
\hline \multicolumn{10}{|l|}{ Cancer $^{\mathrm{e}}$} \\
\hline No & 1977 & 3.61 & 0.56 & & & 0.831 & 0.013 & & \\
\hline Yes & 401 & 5.21 & 0.31 & 1.60 & 0.64 & 0.805 & 0.010 & 0.025 & 0.016 \\
\hline \multicolumn{10}{|c|}{ No. of conditions } \\
\hline 0 & 301 & 3.42 & 0.33 & & & 0.884 & 0.010 & & \\
\hline 1 & 613 & 2.66 & 0.47 & 0.76 & 0.57 & 0.859 & 0.013 & 0.025 & 0.017 \\
\hline 2 & 671 & 3.14 & 0.21 & 0.28 & 0.39 & 0.853 & 0.006 & 0.031 & 0.012 \\
\hline $3+$ & 795 & 5.60 & 1.74 & 2.18 & 1.77 & 0.751 & 0.032 & 0.133 & 0.034 \\
\hline
\end{tabular}

${ }^{a}$ Mortality rate per 100 person-years of follow-up, adjusted by age and sex in subgroups

${ }^{b}$ Standard error

${ }^{c}$ EQ5D index, adjusted by age and sex in subgroups

${ }^{\mathrm{d}}$ Coronary heart disease, angina, or heart attack

e Any kind of cancer, excluding skin cancer (0.075-point or $8.7 \%$ lower). Overall, stroke was associated with more than half $(52.6 \%)$ of the QALY loss or a loss of 7.8 years in QALY. Such a large stroke-associated QALY loss also occurred for both men (5.6-year loss) and women (9.8-year loss) and for white non-Hispanics (8.6-year loss) and black non-Hispanics (5.4-year loss).

\section{Loss due to emphysema or asthma}

In this population, $6.2 \%$ of participants had emphysema and $7.5 \%$ of participants reported that they had asthma at the time of their interview. Emphysema had both a large impact on a participant's mortality and HRQOL. The mortality rate, adjusted by age and sex, among those with emphysema was about threefold of that among those without emphysema (10.55 vs. 3.42). The EQ-5D index among participants with emphysema was $8.9 \%$ lower than that among participants without emphysema (0.758 vs. 0.832). Overall, emphysema had a very large impact on QALY with a loss of 9.5 years $(64.2 \%)$ in QALY and this 9.5-year loss was the most of the nine diseases examined. The QALY loss to emphysema occurred for both men (7.8- 
Table 3 Quality-adjusted life years (QALY) by diseases status and QALY loss due to each disease

\begin{tabular}{|c|c|c|c|c|c|}
\hline Diseases & QALY $^{\mathrm{a}}$ & $\mathrm{SE}^{\mathrm{b}}$ & $\operatorname{Loss}^{c}$ & SE & $\%$ Loss \\
\hline Total & 13.7 & 1.3 & - & - & - \\
\hline \multicolumn{6}{|c|}{ Depression } \\
\hline No & 14.1 & 1.5 & & & \\
\hline Yes & 4.6 & 1.5 & 9.4 & 1.4 & 67.1 \\
\hline \multicolumn{6}{|l|}{ Diabetes } \\
\hline No & 15.3 & 1.5 & & & \\
\hline Yes & 9.0 & 1.4 & 6.3 & 1.4 & 41.2 \\
\hline \multicolumn{6}{|c|}{ Hypertension } \\
\hline No & 15.5 & 1.4 & & & \\
\hline Yes & 13.4 & 1.7 & 2.1 & 1.8 & 13.3 \\
\hline \multicolumn{6}{|c|}{ Heart disease ${ }^{\mathrm{d}}$} \\
\hline No & 16.0 & 1.4 & & & \\
\hline Yes & 9.5 & 1.2 & 6.5 & 1.6 & 40.7 \\
\hline \multicolumn{6}{|l|}{ Stroke } \\
\hline No & 14.8 & 3.2 & & & \\
\hline Yes & 7.0 & 1.3 & 7.8 & 2.9 & 52.6 \\
\hline \multicolumn{6}{|c|}{ Emphysema } \\
\hline No & 14.8 & 2.4 & & & \\
\hline Yes & 5.3 & 1.1 & 9.5 & 2.9 & 64.2 \\
\hline \multicolumn{6}{|l|}{ Asthma } \\
\hline No & 14.2 & 1.5 & & & \\
\hline Yes & 9.6 & 1.4 & 4.6 & 1.6 & 32.3 \\
\hline \multicolumn{6}{|l|}{ Arthritis } \\
\hline No & 13.9 & 2.0 & & & \\
\hline Yes & 13.5 & 0.9 & 0.4 & 3.1 & 3.1 \\
\hline \multicolumn{6}{|l|}{ Cancer $^{\mathrm{e}}$} \\
\hline No & 14.4 & 3.8 & & & \\
\hline Yes & 10.7 & 2.2 & 3.6 & 1.8 & 25.4 \\
\hline \multicolumn{6}{|c|}{ No. of conditions } \\
\hline 0 & 22.9 & 1.4 & & & \\
\hline 1 & 17.4 & 1.0 & 5.5 & 3.0 & 23.8 \\
\hline 2 & 13.7 & 1.5 & 9.2 & 3.7 & 40.3 \\
\hline $3+$ & 10.3 & 0.8 & 12.6 & 1.5 & 55.1 \\
\hline
\end{tabular}

${ }^{a}$ Mean QALY throughout the remainder of the lifetime, adjusted for age and sex for subgroups

b Standard error

${ }^{c}$ Loss in QALY throughout the remainder of the lifetime

d Coronary heart disease, angina, or heart attack

e Any kind of cancer, excluding skin cancer

year loss) and women (10.6-year loss) and for white nonHispanics (10.0-year loss) and black non-Hispanics (6.4year loss). The loss for Hispanics was also large (7.3-year loss), but it was not statistically significant, probably due to small sample size $(n=10)$.

Asthma had a large impact on mortality but a relatively smaller impact on HRQOL: a 2.38-point increase (71.7 \%) in mortality rate and a 0.080 -point decrease $(9.6 \%)$ in EQ-
5D index. Overall, asthma was associated with a relatively large amount to the QALY loss, with a loss of 4.8 years (37.2\%). In subgroups, the loss was statistically significant for men only (4.9-year loss).

\section{Loss due to arthritis}

More than half $(55.5 \%)$ participants had arthritis. However, the health impact of arthritis was very small for both mortality and HRQOL. The QALY was nearly the same for those with arthritis and for those without arthritis (13.5 vs. 13.9 years).

\section{Loss due to cancer}

About $17.6 \%$ of participants reported having cancer. Compared those without cancer, participants with cancer had a relatively large increased mortality rate, an increase of 1.69 points $(44.3 \%)$. However, their EQ-5D index was nearly the same ( 0.805 vs. 0.831$)$. Overall, the QALY loss due to cancer was 3.6 years and such a loss was statistically significant. In subgroups, only women (7.1-year loss) and white non-Hispanics (3.9-year loss) had a statistically significant QALY loss due to cancer.

\section{Loss due to number of chronic conditions}

Participants had an average of 2.0 chronic conditions $(\mathrm{SD}=1.3)$. Nearly nine in ten $(87.8 \%)$ participants had at least one chronic condition, and about one-third (33.1\%) had at least three chronic conditions. Compared to those without any of the nine chronic conditions, participants with one condition had an associated loss of 5.5 years in QALY; those with two conditions, a loss of 9.2 years in QALY; and those with three or more conditions, a loss of 12.6 years in QALY.

\section{Discussion}

This study estimated mean QALY throughout the remainder of the lifetime for participants aged 65 and older from the NHANES-Linked Mortality File for nine chronic conditions. This method estimates mean QALY based on each participant's observed HRQOL score, mortality status, and follow-up time and, therefore, differs from the life table method which uses the "group average" of mortality rates and HRQOL scores in different age intervals $[12,18]$. This method has several advantages over previous methods. First, it does not rely on mortality estimates for the target population and so allows for the calculation of QALY loss for any diseases and risk factors included in the NHANES. Second, it can account for the strong association 

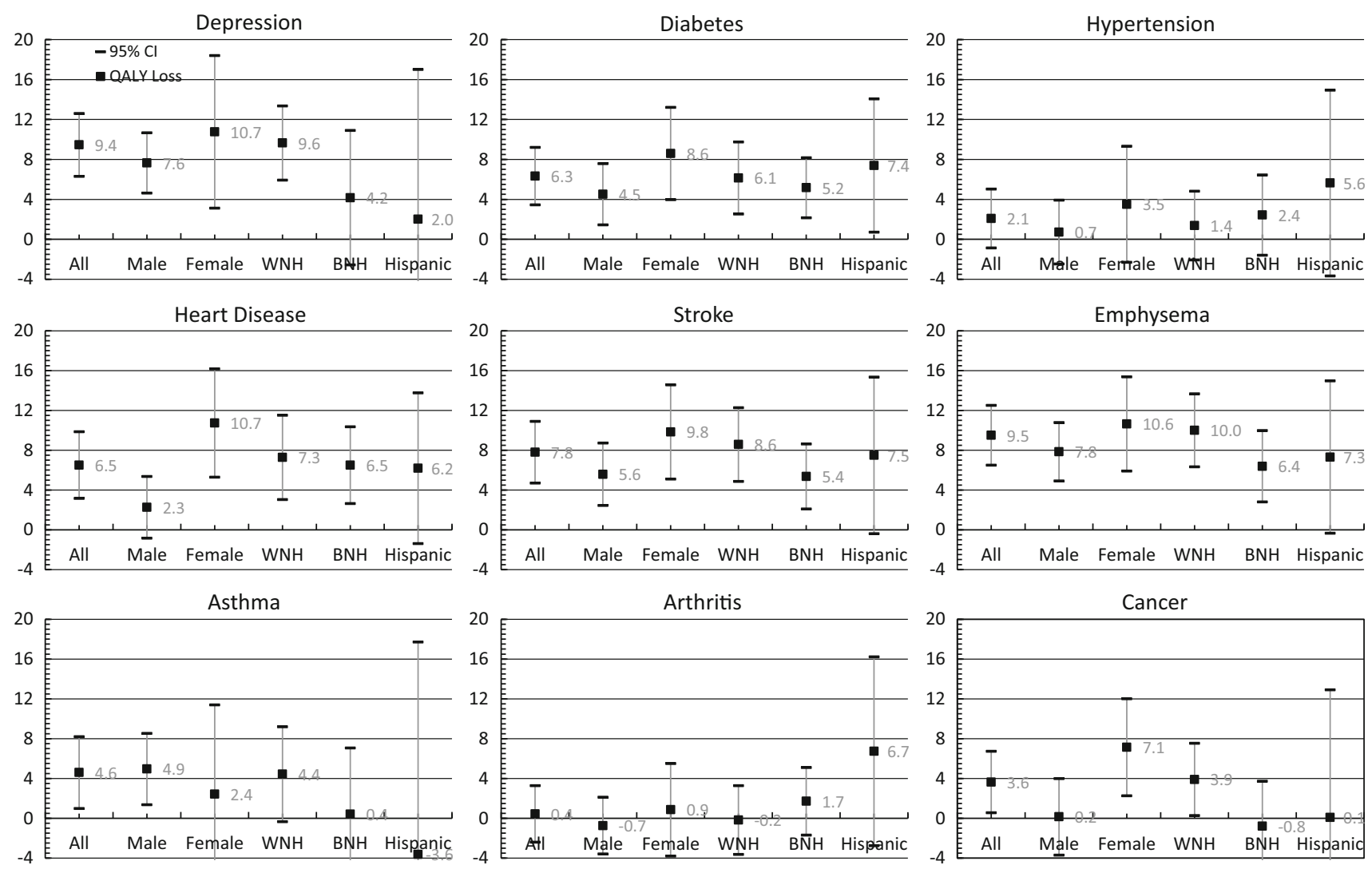

WNH: white non-Hispanics

BNH: black non-Hispanics

Fig. 1 QALY loss due to each disease, overall, by sex, and by race/ethnicity

between HRQOL and survival time at the individual level for the variance estimation, and, therefore, this method requires smaller sample sizes. Our analyses showed that QALY estimates were relatively robust even with a small sample size of approximately 100. Third, it can account for differences in participants' characteristics between the different subgroups, and, as a result, can adjust for any confounding variables for the comparison of mean QALY between diseased and nondiseased persons.

In this study, depression and emphysema were associated with the most overall health losses for US elderly with more than $60 \%$ losses in QALY, followed by stroke which was associated with more than $50 \%$ QALY loss. Diabetes and heart disease were associated with approximately $40 \%$ QALY losses. Asthma was associated with a little over $30 \%$ QALY loss, and cancer was associated with about $25 \%$ QALY loss. Finally, hypertension had a small health loss with just a $12 \%$ loss in QALY and arthritis had a negligible QALY loss with a loss of QALY by only $3.1 \%$. In general, these findings were consistent with our understanding of these diseases and with findings of DALY for these conditions from the Global Burden of Disease Study $[8,32]$ and QALE loss due to selected chronic conditions calculated based on the life table method [17, 18, 33].
However, in this study, cancer was associated with only 3.6 QALY loss, a magnitude smaller than the losses to the following six conditions: depression, emphysema, stroke, heart disease, diabetes, and asthma. This loss was smaller than the previously estimated health losses due to cancer [8]. For example, the Global Burden of Disease Study found that cancer was associated with significantly more DALY than the other six conditions [8]. There are at least three explanations for our different results. First, the DALY for cancer was calculated for all ages, so many DALYs were associated with younger persons with faster growing cancers. For persons aged 65 years and older, the most common type of cancer is prostate cancer for men and breast cancer for women, both of which have higher survival rates [34]. Second, because of current cancer screening guidelines and terminology, prostate and breast cancer, as well as other cancers such as thyroid cancer, may be subject to overdiagnosis and overtreatment [35]. Third, many participants in this study who reported cancer had lived with cancer for many years. Specifically, the average number of years since been told of having cancer was 11.9 years and $67 \%$ of participants with cancer reported having cancer for five or more years. Therefore, many cancer patients in this study were cancer survivors and some of them were "free of" cancer at the time of the interview. 
Diseases with approximately the same number of QALY loss may have different losses to fatal and nonfatal outcomes. For example, depression and emphysema had nearly the same QALY loss. Depression lost a much larger proportion of QALY to poor HRQOL score while emphysema lost a much larger portion of QALY to high mortality. Emphysema has been associated with a higher mortality rate [36] while depression has been demonstrated to have a marked impact on morbidity [37].

We calculated QALY loss due to each of nine selected chronic diseases as the decrease in QALY for those with the disease as compared to those without the disease. Of note, participants without a certain disease might have other diseases. For example, in our data, among participants without depression, $87.3 \%$ had at least one of the other eight diseases. Also, having one disease might be associated with an increased risk of having some other diseases [38]. Depression has been associated with an increased risk of heart disease [39], and, for this sample, the odds ratio was 2.1. One of the solutions for this problem is to calculate QALY loss due to a disease by adjusting for the number of other diseases. After adjusting for the difference in the number of other diseases as well as age and sex, the QALY loss due to asthma decreased from 4.6 to 3.1 years. For the other eight conditions, the QALY losses were mostly unchanged and still in a similar rank order of QALY loss as the QALY not adjusted by the number of comorbid conditions.

There were several weaknesses for this study. First, respondents reported their own disease status, which was not validated by medical chart reviews. However, the likelihood is small that respondents would report having a disease that they did not have, as opposed to omitting to note having been diagnosed with a given disease. Moreover, because persons who have diabetes or hypertension may not be aware of having this condition, this study may have underestimated the disease prevalence and thus the losses in QALY due to these diseases. Second, the PHQ-8 is not a clinical diagnostic tool for diagnosing depression, but has been used primarily as a screening instrument for estimating the prevalence of depression in the general population. Third, the NHANES did not include the preference-based HRQOL questions. We used a mapping algorithm to obtain EQ-5D index scores for respondents based their answers to the four healthy days questions. Therefore, estimates of QALY loss would also likely be underestimated due to regression toward the mean [18]. However, a previous study examined the bias of QALE estimates and showed that these underestimations were about $2.5 \%$ for QALE loss [18]. Fourth, this method requires a sufficient number of deaths during the follow-up in order to obtain a reliable estimation of QALY. Therefore, this method cannot provide a reliable QALY estimation for a younger population or groups with lower mortality unless the follow-up period is sufficiently long.

This study presents a novel method to estimate the burden of disease for persons affected by each of nine common chronic conditions as well as the impact of having one or more conditions by calculating mean QALYs throughout the remainder of the lifetime. QALY encompasses both years of life lost and relative severity of the disease and can be used to ascertain the cost-effectiveness of alternative opportunities to reduce the health burden of a risk factor or particular disease [5, 6]. While the method is novel, it has the potential to provide estimates of the burden of diseases for a wide range of risk factors and chronic health conditions in target populations (i.e., elderly or "high-risk" subgroups) in order to improve population health. The method developed here may also be applied to a randomized controlled trial to compare lifetime health outcomes between treatment and control groups. These analyses might help inform clinical practice and guide community health promotion efforts relating to the potential impact of risk factors and conditions on a given person as well as population subgroups. In particular, this method can be used to estimate the contributions of specific diseases to socioeconomic disparities in QALY. Future research should focus on the relationship between clusters of conditions and associated behavioral risk factors and QALY loss as well as the application of these methods to other data sets which contain more detailed information pertaining to the diagnosis of clinical conditions.

\section{Compliance with ethical standards}

Conflict of interest The authors declare that there is no conflict of interests regarding the publication of this paper.

Ethical statements This analysis used de-identified data produced by federal agencies in the public domain. Data were downloaded from the Centers for Disease Control and Prevention Website (ftp://ftp.cdc. gov/pub).

\section{References}

1. Institute of Medicine. (2012). Living well with chronic illness: A call for public health action. Washington, DC: The National Academies Press.

2. Roglic, G., \& Unwin, N. (2010). Mortality attributable to diabetes: Estimates for the year 2010. Diabetes Research and Clinical Practice, 87(1), 15-19.

3. Holmes, J., McGill, S., Kind, P., Bottomley, J., Gillam, S., \& Murphy, M. (2000). Health-related quality of life in type 2 diabetes (TARDIS-2). Value in Health, 3(Suppl 1), 47-51.

4. Field, M. J., \& Gold, M. R. (Eds.). (1998). Summarizing population health: Directions for the development and application of population metrics. Washington, DC: Institute of Medicine, National Academy Press. 
5. Gold, M. R., Siegel, J. E., Russell, L. B., \& Weinstein, M. C. (Eds.). (1996). Cost-effectiveness in health and medicine. New York: Oxford University Press.

6. Brown, D. S., Jia, H., Zack, M. M., Thompson, W. W., Haddix, A. C., \& Kaplan, R. M. (2013). Using health-related quality of life and quality-adjusted life expectancy for effective public health surveillance and prevention. Expert Review of Pharmacoeconomics and Outcomes Research, 13(4), 425-427.

7. Gold, M. R., Stevenson, D., \& Fryback, D. G. (2002). HALYS and QALYS and DALYS, Oh My: Similarities and differences in summary measures of population health. Annual Review of Public Health, 23, 115-134.

8. Murray, C. J., Vos, T., Lozano, R., Naghavi, M., Flaxman, A. D., Michaud, C., et al. (2012). Disability-adjusted life years (DALYs) for 291 diseases and injuries in 21 regions, 1990-2010: A systematic analysis for the global burden of disease study 2010. Lancet, 380(9859), 2197-2223.

9. Sullivan, D. (1971). A single index of mortality and morbidity. HSMHA Health Reports, 86, 347-354.

10. Chiang, C. L. (1984). Statistical inference regarding life table functions. In C. L. Chiang (Ed.), The life table and its applications (pp. 153-167). Malabar, FL: Robert E. Krieger Publishers.

11. National Research Council. (2010). Accounting for health and health care: Approaches to measuring the sources and costs of their improvement. Panel to Advance a research program on the Design of National Health Accounts, Committee on National Statistics, Division of Behavioral and Social Sciences and Education. Washington, DC: The National Academies Press.

12. Jia, H., Zack, H. H., \& Thompson, W. W. (2011). State qualityadjusted life expectancy for US adults from 1993 to 2008. Quality of Life Research, 20(6), 853-863.

13. Glasziou, P. P., Simes, R. J., \& Gelber, R. D. (1990). Quality adjusted survival analysis. Statistics in Medicine, 9, 1259-1276.

14. Shen, L. Z., Pulkstenis, E., \& Moseyni, M. (1999). Estimation of mean quality adjusted survival time. Statistics in Medicine, 18, $1541-1554$

15. Gong, Q., \& Fang, L. (2012). Asymptotic properties of mean survival estimate based on the Kaplan-Meier curve with an extrapolated tail. Pharmaceutical Statistics, 11, 135-140.

16. Stewart, S. T., Cutler, D. M., \& Rosen, A. B. (2013). US trends in quality-adjusted life expectancy from 1987 to 2008: Combining national surveys to more broadly track the health of the nation. American Journal of Public Health, 103(11), e78-e87.

17. Jia, H., Zack, M. M., \& Thompson, W. W. (2013). The effects of diabetes, hypertension, asthma, heart disease, and stroke on quality-adjusted life expectancy. Value in Health, 16(1), 140-147.

18. Jia, H., Zack, M. M., Thompson, W. W., \& Dube, S. R. (2013). Quality-adjusted life expectancy (QALE) loss due to smoking in the United States. Quality of Life Research, 22(1), 27-35.

19. Lee, H. Y., Hwang, J. S., Jeng, J. S., \& Wang, J. D. (2010). Quality-adjusted life expectancy (QALE) and loss of QALE for patients with ischemic stroke and intracerebral hemorrhage: A 13-year follow-up. Stroke, 41(4), 739-744.

20. Oyunbileg, S., Wang, J. D., Sumberzul, N., Chang, Y. Y., \& Erdenchimeg, E. (2011). Health impact of pneumoconiosis in Mongolia: Estimation of losses in life expectancy and quality adjusted life expectancy. American Journal of Indian Medicine, 54(4), 285-292.

21. Richardson, G., \& Manca, A. (2004). Calculation of quality adjusted life years in the published literature: A review of methodology and transparency. Health Economics, 12, 1203-1210.

22. Ong, K. L., Wu, B. J., Cheung, B. M., Barter, P. J., \& Rye, K. A. (2013). Arthritis: Its prevalence, risk factors, and association with cardiovascular diseases in the United States, 1999 to 2008. Annals of Epidemiology, 23(2), 80-86.

23. Heron, M. (2015). Deaths: Leading causes for 2011. National Vital Statistics Reports, 64(7), 1-96.
24. U.S. Burden of Disease Collaborators. (2013). The state of US health, 1990-2010: Burden of diseases, injuries, and risk factors. JAMA, 310(6), 591-608.

25. Centers for Disease Control and Prevention (CDC). National Center for Health Statistics (NCHS). National Health and Nutrition Examination Survey Data. Hyattsville, MD: U.S. Department of Health and Human Services, Centers for Disease Control and Prevention, 2005-2006. http://wwwn.cdc.gov/nchs/ nhanes/search/nhanes05_06.aspx. Accessed 20 July 2015.

26. Centers for Disease Control and Prevention (CDC). National Center for Health Statistics (NCHS). NCHS Data Linked to Mortality Files. Hyattsville, MD: U.S. Department of Health and Human Services, Centers for Disease Control and Prevention, 2011. http://www.cdc.gov/nchs/data_access/data_linkage/mortal ity.htm. Accessed 20 July 2015.

27. Centers for Disease Control and Prevention. (2000). Measuring healthy days: Population assessment of health-related quality of life. U.S. Department of Health and Human Services. Centers for Disease Control and Prevention. National Center for Chronic Disease Prevention and Health Promotion. Division of Adult and Community Health. http://www.cdc.gov/hrqol/pdfs/mhd.pdf. Accessed 20 July 2015.

28. Jia, H., \& Lubetkin, E. I. (2008). Estimating EuroQol EQ-5D scores from population healthy days data. Medical Decision Making, 28(4), 491-499.

29. Jia, H., Zack, M. M., Moriarty, D. G., \& Fryback, D. G. (2011). Predicting the EuroQol Group's EQ-5D Index from CDC's "Healthy Days" in a US sample. Medical Decision Making, 31(1), 174-185.

30. Kroenke, K., Strine, T. W., Spitzer, R. L., Williams, J. B., Berry, J. T., \& Mokdad, A. H. (2009). The PHQ-8 as a measure of current depression in the general population. Journal of Affective Disorders, 114(1-3), 163-173.

31. Austin, P. C. (2011). An introduction to propensity score methods for reducing the effects confounding in observational studies. Multivariate Behavioral Research, 46(3), 399-424.

32. Ferrari, A. J., Charlson, F. J., Norman, R. E., Patten, S. B., Freedman, G., \& Murray, C. J. (2013). Burden of depressive disorders by country, sex, age, and year: Findings from the global burden of disease study. PLoS Medicine, 10(11), e1001547.

33. Jia, H., Zack, M. M., Thompson, W. W., Crosby, A. E., \& Gottesman, I. I. (2015). Impact of depression on quality-adjusted life expectancy (QALE) directly as well as indirectly through suicide. Social Psychiatry and Psychiatric Epidemiology, 50(6), 939-949.

34. American Cancer Society. Cancer Facts \& Figures 2015. Atlanta: American Cancer Society; 2015. http://www.cancer.org/acs/ groups/content/@editorial/documents/document/acspc-044552.pdf. Accessed on 20 July 2015.

35. Ford, E. S. (2014). Trends in mortality from chronic obstructive pulmonary disease among adults in the United States. Chest,. doi:10.1378/chest.14-2311.

36. Welch, H. G., \& Black, W. C. (2010). Overdiagnosis in cancer. Journal of the National Cancer Institute, 102, 605-613.

37. Vos, T., Flaxman, A. D., Naghavi, M., Lozano, R., Michaud, C., Ezzati, M., et al. (2012). Years lived with disability (YLDs) for 1160 sequelae of 289 diseases and injuries 1990-2010: A systematic analysis for the global burden of disease study 2010 . Lancet, 380(9859), 2163-2196.

38. Vogeli, C., Shields, A. E., Lee, T. A., Gibson, T. B., Marder, W. D., Weiss, K. B., et al. (2007). Multiple chronic conditions: Prevalence, health consequences, and implications for quality, care management, and costs. Journal of General Internal Medicine, 22(Suppl 3), 391-395.

39. Seligman, F., \& Nemeroff, C. B. (2015). The interface of depression and cardiovascular disease: Therapeutic implications. Annals of the New York Academy of Science, 1345(1), 25-35. 